3 Antithrombotic Trialists' Collaboration: Collaborative meta-analysis of randomised trials of antiplatelet therapy for prevention of death, myocardial infarction, and stroke in high risk patients. BMJ 2002;324: 71-86.

4 Algra A, van Gijn J, Koudstaal PJ: Secondary prevention after cerebral ischaemia of presumed arterial origin: is aspirin still the touchstone? J Neurol Neurosurg Psychiatry 1999;66:557-559.

5 European Stroke Prevention Study 2: efficacy and safety data. J Neurol Sci 1997;151(suppl):S1-S77.

6 Dresse A, Chevolet C, Delapierre D, Masset H, Weisenberger H, Bozler G, Heinzel G: Pharmacokinetics of oral dipyridamole (Persantine) and its effect on platelet adenosine uptake in man. Eur J Clin Pharmacol 1982;23:229-234

7 Derendorf H, Vander Maelen CP, Brickl RS, MacGregor TR, Eisert W: Dipyridamole bioavailability in subjects with reduced gastric acidity. J Clin Pharmacol 2005;45:845-850.

Hartmut Derendorf, PhD

Distinguished Professor and Chair, Department of Pharmaceutics

University of Florida, 100494, College of Pharmacy

Gainesville, FL 32610 (USA)

Tel. +1 352846 2726, Fax +1 3523923249

E-Mail Hartmut@cop.ufl.edu

Cerebrovasc Dis 2007;23:73-74

DOI: $10.1159 / 000097033$

\section{Reply to the Letter by Derendorf: Clinical Trials and Dipyridamole Formulation Selection}

G. Grieco, S. Jonas

Section of Quantitative Investigation, Department of Neurology, New York University School of Medicine, New York, N.Y., USA

Dr. Derendorf has offered a detailed critique of our editorial, and raised some interesting questions. After careful consideration, we must disagree with some of his observations and comments. In particular, we note:

(1) Dr. Derendorf quotes us as having stated that 'antiplatelet therapy with aspirin plus extended-release dipyridamole is the best treatment yet studied for secondary stroke prevention after noncardioembolic stroke'. However, we stated that 'antiplatelet therapy with dipyridamole plus aspirin is the best treatment yet studied for secondary stroke prevention after non-cardioembolic stroke' (emphases added).

(2) Dr. Derendorf states that 'Jonas and Grieco argue that the only remaining question from stroke prevention trials is "which formulation of dipyridamole should be employed"'. We would be foolhardy or naïve to believe that would be the only unanswered question; we are well aware of many other unresolved issues regarding stroke prevention. What we did say was that given that the best available evidence favors treatment with dipyridamole plus aspirin, what formulation of dipyridamole should be used?

(3) Dr. Derendorf argues that '... the only trials that showed clinical benefit from aspirin plus dipyridamole combined treat- ment used extended-release formulations in doses of $200 \mathrm{mg}$ twice daily rather than conventional immediate-release tablets that are recommended by Jonas and Grieco'. We are surprised by this conclusion. Our table shows the results of the AICLA and ESPS1 trials of conventional dipyridamole plus aspirin versus placebo. The relative risks of the individual trials and of the metaanalysis (0.581, 0.620 and 0.614 , respectively; all statistically significant) are remarkably similar to the relative risk (0.628) of extended-release dipyridamole plus aspirin versus placebo in the ESPS2 study.

(4) Dr. Derendorf states: 'A separate meta-analysis of 4 early studies in a total of 1,757 patients that examined effects of conventional dipyridamole found only a $3 \%$ added benefit from dipyridamole compared with aspirin that was not significant.' He does not explain that the endpoint was not stroke but a triple endpoint including stroke. He also does not indicate that one of the studies used a 150-mg total daily dipyridamole regimen, which is not a regimen for which we make a clinical claim, and not a regimen we included in our analyses. Furthermore, the reference for one of the studies is an unpublished 'Boehringer Ingelheim internal report'.

(5) We are baffled by the statement 'dipyridamole dose selection of a 3 times daily 75-mg tablet appears arbitrary'. To quote the ESPS2 Study Group in the introduction to the report of their study results: 'In 1987 the first ESPS study found that a combination of ASA (990 mg daily) and dipyridamole (225 mg daily) compared to placebo achieved the largest stroke risk reduction ever reported for stroke and TIA patients (38.1\%)' [1]. Our interest in a 225 -mg daily dipyridamole regimen is based on this important study sponsored, as was ESPS2, by Boehringer Ingelheim.

(6) On the question of headache, it would be nice to have the dose- and duration-related discontinuation data Dr. Derendorf asks for. Publicly available sources do not provide data from the ESPS1 trial on treatment discontinuations due to headache and other adverse events. For ESPS2 the discontinuation rates in the scientific literature and the product labeling do not agree. Thus, the ESPS2 group in their safety paper [2] reported that $8.0 \%$ of subjects on extended-release dipyridamole (with or without aspirin) dropped out because of headache, versus $2.1 \%$ of subjects on aspirin or placebo $(\mathrm{p}<0.001)$. The FDA-approved product labeling reports (in its table 3 ) discontinuation rates of $10 \%$ for both the Aggrenox ${ }^{\circledR}$ and extended-release dipyridamole groups, $3 \%$ for the aspirin group and $4 \%$ for the placebo group.

We stand by our comments on headaches. We made it clear that what we were saying was based on our clinical experience. This experience is consistent with the reported severalfold higher peak plasma concentrations attained with the extended-release formulation (see item 7a below) compared with a conventional formulation. It is also consistent with Boehringer Ingelheim's own conclusions on the association of headache severity and duration with plasma concentrations of dipyridamole [3].

(7) In support of the claimed superiority of extended-release versus conventional formulations, Dr. Derendorf cites the article he wrote [4], with 4 co-authors from Boehringer Ingelheim, comparing dipyridamole pharmacokinetics for Aggrenox and generic dipyridamole in 42- to 64-year-old normal subjects whose gastric $\mathrm{pH}$ was purposely raised to at least 4 by administration of a proton pump inhibitor. Ultimately, bioavailability differences do not matter when there is no difference in efficacy demonstrated in controlled trials. It is nonetheless interesting to note that while 
he showed (in his table 1 and fig. 1) the dipyridamole $\mathrm{AUC}_{0-12 \mathrm{~h}}$ and $\mathrm{C}_{\max }$ for the generic to be about half those for the extendedrelease form:

(a) there was much less peak-to-trough variability for the generic than for Aggrenox (a ratio of approx. 2:1 vs. $\sim 6: 1$ );

(b) the generic had equal or greater serum concentrations after about $9 \mathrm{~h}\left(\mathrm{C}_{12} \mathrm{~h}\right.$ about 1.5 times that for Aggrenox) and an $\mathrm{AUC}_{0-48 \mathrm{~h}}$ about three quarters that of Aggrenox;

(c) the lower AUCs and $\mathrm{C}_{\max }$, for the generic, comparable to the effects of high-fat meals on the kinetics of Aggrenox, may not result in clinically relevant decreases in adenosine uptake inhibition in platelets, erythrocytes and endothelial cells [5].

It is also interesting to note that 'in ESPS2 ... plasma concentrations (determined as AUC) of dipyridamole in healthy elderly subjects ( $>65$ years) were about $40 \%$ higher than in subjects younger than 55 years receiving treatment with Aggrenox' [6]. This may counteract the effects of decreased acidity, as may be seen with concomitant proton pump inhibitor therapy, which is more commonly encountered in the older population.

We thank Dr. Derendorf for providing us this opportunity to address the issues raised in his letter.

\section{References}

1 European Stroke Prevention Study 2: efficacy and safety data. 1. Introduction. J Neurol Sci 1997;151(suppl):S1-S2.

2 European Stroke Prevention Study 2: efficacy and safety data. 8. Safety. J Neurol Sci 1997;151(suppl):S41-S51

3 Theis JG, Deichsel G, Marshall S: Rapid development of tolerance to dipyridamole-associated headaches. Br J Clin Pharmacol 1999;48:750755.

4 Derendorf H, Vander Maelen CP, Brickl RS, MacGregor TR, Eisert W: Dipyridamole bioavailability in subjects with reduced gastric acidity. J Clin Pharmacol 2005;45:845-850.

5 Aggrenox $^{\circledR}$ (aspirin/extended-release dipyridamole) US Product Labeling, subsection: Clinical Pharmacology, Pharmacokinetics, Dipyridamole, Effect of Food.

6 Aggrenox $^{\circledR}$ (aspirin/extended-release dipyridamole) US Product Labeling, subsection: Clinical Pharmacology, Special Populations, Geriatric Patients.

Saran Jonas, MD

Section of Quantitative Investigation, Department of Neurology New York University School of Medicine

550 First Avenue, NBV 7W-11, New York, NY 10016 (USA)

Tel. +1 212263 7591, Fax +1 2122638228

E-Mailsaran.jonas@med.nyu.edu 